\title{
Das Parkinson-Syndrom in der allgemeinmedizinischen Praxis
}

Reinhold Glehr, Tobias Schöberl, Werner Seel

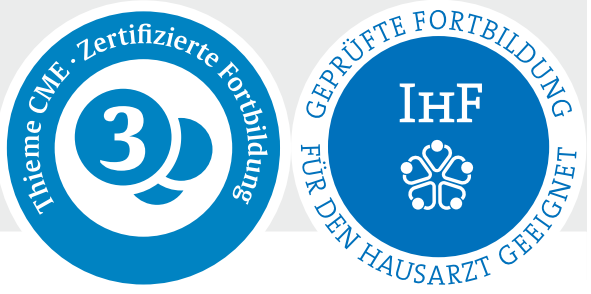

\begin{abstract}
Die Begleitperson berichtet Ihnen unspezifische Symptome, die sich nur langsam verändern und vom Betroffenen gar nicht wahrgenommen werden. In anderen Fällen könnte ein essenzieller Tremor vorliegen, oder es ist eine medikamentös induzierte Parkinson-Symptomatik zu bedenken, oder Sie sind mit der psychosozialen Situation des Erkrankten konfrontiert. Dieser Beitrag zeigt Ihnen, wie Sie diagnostisch und therapeutisch adäquat handeln können.
\end{abstract}

\section{Frühsymptomatik}

Wie viele andere Patienten und Patientinnen in der allgemeinmedizinischen Praxis weisen jene mit Morbus Parkinson oder anderen akinetisch-rigiden Syndromen im Frühstadium oft unspezifische und vielfältige Symptome auf. Da die Veränderungen meist allmählich erfolgen, entgehen diese der Aufmerksamkeit bei Routinekonsultationen. Oft sind es Partner oder Partnerin, die mitkommen und die Veränderungen thematisieren, da die Betroffenen zu wenig Selbstwahrnehmung haben. Möglichst früh die richtige Diagnose zu stellen gestaltet sich daher oft als schwierig, erfordert eine aufmerksame Anamnese-Erhebung, eine sorgfältige klinische Untersuchung sowie die frühe Zusammenarbeit mit Fachärzten/-innen für Neurologie.

\section{Prävalenz, Ätiologie, Pathogenese, Mortalität}

Parkinson-Syndrome stellen nach Demenz die häufigsten neurodegenerativen Erkrankungen in der allgemeinmedizinischen Praxis dar. Die Prävalenz beträgt $0,15 \%$, steigt jedoch mit zunehmendem Alter an: bei den $>60$-Jährigen auf $1 \%$ und bei den $>80$-Jährigen auf $3 \%$ [1]. Männer sind i.d.R. in gleicher Häufigkeit betroffen wie Frauen.

Die häufigste Form ist das idiopathische Parkinson-Syndrom, die Ätiologie der Erkrankung also unklar. In 5$15 \%$ werden hereditäre Parkinson-Erkrankungen beobachtet. Vor allem bei Patienten/-innen mit einer Krankheitsmanifestation vor dem 45. Lebensjahr sowie mindestens zwei Verwandten 1. Grades mit ähnlicher Symptomatik sollte daran gedacht werden. Weiterhin werden atypische und sekundäre Parkinson-Syndrome unterschieden (siehe hierzu auch $>$ Tab. 1).

\section{FALLBEISPIEL 1}

Frühsymptomatik

Eine 68-jährige Patientin, die schon lange bei Ihnen in Betreuung wegen Hypertonie steht, konsultiert Sie in Ihrer Praxis. Heute wolle sie einmal über etwas ganz anderes mit Ihnen reden. Sie ist Schriftführerin in einem Verein, in letzter Zeit falle ihr das Schreiben aber immer schwerer, was sie sehr belaste. Sie sei nicht mehr so schnell wie davor. Außerdem sei sie in letzter Zeit ungeschickter als früher, ein paar Mal sei sie gestolpert und gestürzt.

Pathogenetisch kommt es zu einer Degeneration dopaminerger Neurone in der Substantia nigra und damit zu einem Dopaminmangel im Striatum. Auch Locus coeruleus, Nucleus basalis, Hypothalamus, cerebraler Kortex, kraniale Motoneuronen sowie zentrale und periphere Anteile des autonomen Nervensystems können von den Veränderungen betroffen sein. Abhängig von den betroffenen Regionen manifestieren sich entsprechende Symptome. In den meisten Fällen sind in den degenerierten Neuronen intrazytoplasmatische neuronale Einschlüsse, sogenannte Lewy-Körperchen zu finden, in denen zahlreiche Proteine angehäuft sind, unter anderem Synuclein und Ubiquitin [2].

\section{DEFINITION}

Als degenerative Erkrankungen werden Krankheitsgeschehen definiert, bei denen Gewebestrukturen und Organe in ihrer Struktur oder Funktion nachhaltig beeinflusst oder geschädigt werden. Die Symptome kommen schleichend und sind gerade in der Frühphase sehr unterschiedlich. 
- Tab. 1 Wichtige Differenzialdiagnosen zum idiopathischen Morbus Parkinson. (Daten aus [1])

\begin{tabular}{|c|c|}
\hline Ursache des Parkinsonismus & Beispiele \\
\hline \multicolumn{2}{|l|}{ Sekundäre Parkinson-Syndrome } \\
\hline $\begin{array}{l}\text { Arteriosklerotischer (oder „vaskulärer“) } \\
\text { Parkinsonismus }\end{array}$ & - z. B. bei zerebraler Mikroangiopathie, bei subkortikaler arteriosklerotischer Enzephalopathie \\
\hline Medikamentös bedingter Parkinsonismus & $\begin{array}{l}\text { - Neuroleptika } \\
\text { " Antiemetika (v. a. Dopaminantagonisten, z. B. Metoclopramid) } \\
\text { - Lithium } \\
\text { - Valproinsäure } \\
\text { - Kalziumantagonisten wie Flunarizin }\end{array}$ \\
\hline Infektiös bedingter Parkinsonismus & $\begin{array}{l}\text { - postenzephalitischer Parkinsonismus (nach Encephalitis lethargica) } \\
\text { " Lues cerebrospinalis } \\
\text { " AIDS-Enzephalopathie }\end{array}$ \\
\hline \multicolumn{2}{|l|}{ Normaldruckhydrozephalus } \\
\hline Toxischer Parkinsonismus & $\begin{array}{l}\text { " CO-Vergiftung } \\
\text { " Mangan-Vergiftung } \\
\text { " MPTP(1-Methyl-4-phenyl-1,2,3,6-tetrahydropyridin, entsteht als Nebenprodukt bei der } \\
\text { Herstellung der Designerdroge MPTP) } \\
\text { " Cyanid } \\
\text { " Methanol }\end{array}$ \\
\hline Trauma & $\begin{array}{l}\text { " wiederholtes stumpfes Trauma, z. B. bei Boxern } \\
\text { " Mittelhirntrauma } \\
\text { " chronisches Subduralhämatom }\end{array}$ \\
\hline Metabolisch & $\begin{array}{l}\text { - Hypo- und Pseudohypoparathyreoidismus mit Basalganglienverkalkungen } \\
\text { - idiopathische Basalganglienverkalkungen, Morbus Fahr }\end{array}$ \\
\hline Weitere Ursachen & $\begin{array}{l}\text { - Tumor } \\
\text { - Polycythaemia vera } \\
\text { - Akanthozytose } \\
\text { - Hemiparkinson-Hemiatrophie-Syndrom, Fragiles-X-Tremor-Ataxie-Syndrom (FXTAS) } \\
\text { " Westphal-Variante der Chorea Huntington } \\
\text { - Prionenerkrankungen }\end{array}$ \\
\hline \multicolumn{2}{|l|}{ Atypische Parkinson-Syndrome } \\
\hline Neurodegenerative Erkrankungen & $\begin{array}{l}\text { - progressive supranukleäre Lähmung } \\
\text { - Multi-System-Atrophien (MSA) } \\
\text { - kortikobasale Degeneration } \\
\text { - Lewy-Körper-Krankheit } \\
\text { - frontotemporale Demenz } \\
\text { - Morbus Alzheimer mit Parkinson-Symptomen }\end{array}$ \\
\hline $\begin{array}{l}\text { Hereditäre Krankheiten, bei denen Parkinson- } \\
\text { Symptome im Vordergrund stehen können }\end{array}$ & $\begin{array}{l}\text { - Morbus Wilson } \\
\text { " Pantothenkinase-assoziierte Neurodegeneration } \\
\text { - SCA3-spinozerebelläre Ataxie }\end{array}$ \\
\hline
\end{tabular}

Die Lebenserwartung hat sich seit Einführung der modernen Morbus Parkinson-Behandlung wesentlich gebessert. Die Studien zur Mortalität ergeben aber sehr unterschiedliche Ergebnisse, vermutlich abhängig von der Genauigkeit der diagnostischen Zuordnung. Nach einer Studie der Universität Innsbruck mit einer Beobachtungszeit über 20 Jahre zeigte sich bis nach 10 Jahre bestehender Krankheitsdauer kein signifikanter Unterschied der Lebenserwartung, aber ein mäßiger Anstieg der Mortalität im weiteren Krankheitsverlauf [3]. Ein erhöhtes Risiko findet sich durch die allgemeine Einschränkung der körperlichen Aktivität, Sturzneigung,
Folgen von Atemwegsinfektionen oder Aspiration wegen einer Schluckstörung.

\section{Erstvorstellung in der allgemeinmedizinischen Praxis}

Viele Frühsymptome, die zum Teil sehr unspezifisch sind, werden von den Betroffenen mit dem natürlichen Alterungsprozess in Zusammenhang gebracht ( Tab.2). Dazu zählen Unruhe, Schlafstörungen, Müdigkeit, depressive Verstimmungen, plötzliche Schweißausbrüche, Darmträgheit. Eine Beeinträchtigung des Geruchssinns tritt häufig Jahre vor den motorischen Zeichen auf. Brennende, ziehende oder krib- 
Tab. 2 Häufige Symptome bei Morbus Parkinson.

\begin{tabular}{|l|l|}
\hline Frühstadium: unspezifisch & im späteren Verlauf \\
\hline - Unruhe (Bewegungs- & - Bradykinese (Bewe- \\
$\begin{array}{l}\text { drang von Armen oder } \\
\text { Beinen) }\end{array}$ & - Gungsverlangsamung) \\
\hline - Schlafstörung & - Sturzeschicklichkeit \\
\hline - Müdigkeit & - Tremor \\
\hline - Schmerzen im Schulter- & - Standunsicherheit \\
gürtel-Armbereich & \\
\hline
\end{tabular}

belnde Schmerzen in Schultern und Armen oder anderen Teilen des Bewegungsapparats können ein Frühsymptom sein, ähneln Beschwerden aus dem orthopädisch-rheumatischen Formenkreis und werden zuerst meist nicht mit einer Parkinson-Krankheit in Verbindung gebracht. Allerdings fallen orthopädisch-degenerative Erkrankungen und Osteoporose altersbedingt oft mit den ersten Parkinson-Frühsymptomen zusammen.

Erst mit dem Fortschreiten der Erkrankung werden die typischen Bewegungsstörungen deutlich. Kennzeichnend ist die Bradykinese. Sie wird beschrieben als Verlangsamung bei der Initiierung und Durchführung willkürlicher Bewegungen bzw. als zunehmende Verlangsamung oder als Abnahme der Amplitude bei repetitiven Bewegungen. An die Diagnose Morbus Parkinson sollte gedacht werden, sobald diese Bewegungsabnahme oder -armut gemeinsam mit mindestens einem der Leitsymptome Ruhetremor, Rigor und/oder Standunsicherheit im Sinne einer posturalen Instabilität (Störung der aufrechten Körperhaltung) vorliegt.

Merke

In der allgemeinmedizinischen Praxis werden die Patienten/-innen oft vordergründig mit motorischen
Symptomen wie Ungeschicklichkeit, Steifheit der Muskulatur oder Zittern vorstellig.

Zudem werden folgende Symptome von den Betroffenen/Angehörigen angegeben:

- Störungen der Feinmotorik,

- Schwierigkeiten beim Schreiben bzw. Veränderung der Schrift (kleiner, unleserlicher; Mikrografie),

- Schwierigkeiten beim Zuknöpfen von Hemd oder Hose,

- Schwäche der Stimme und

- häufig eine ungewöhnlich rasche Alterung.

In späteren Stadien treten oft Schluckstörungen in den Vordergrund der Beratung.

Häufig werden zunächst ausschließlich oder hauptsächlich einseitige Beschwerden im Bewegungsapparat beschrieben, die sich allmählich auf beide Seiten ausdehnen. Differenzialdiagnostisch zum idiopathischen Morbus Parkinson ist ein arzneimittelinduziertes Parkinson-Syndrom immer von Anfang an beidseitig. Auf Nachfrage sind zum Zeitpunkt der Erstvorstellung wegen motorischer Symptome oft die in der $>$ Tab. 3 genannten nichtmotorischen Symptome bereits seit längerem vorhanden.

Merke
Unspezifische Symptome wie Schlafstörungen, Obs-
tipation oder Geruchsstörungen können als Anzei-
chen der fortschreitenden Hirnerkrankung schon
Jahre vor den motorischen Hauptsymptomen auf-
treten.

- Tab. 3 Nichtmotorische Symptome des Morbus Parkinson.

\begin{tabular}{|l|l|l|}
\hline Störung der autonomen Funktionen & Kognitive Symptome & Emotionale/psychiatrische Symptome \\
\hline - Hyperhidrose & " verlangsamtes Denken (Bradyphrenie) & - Depression \\
\hline - Hypersalivation & - Perseveration, krankhaftes Beharren & - Apathie \\
\hline - Seborrhoe & - gestörte Handlungsplanung und & - Angst \\
\hline - Obstipation & -ausführung & - Halluzinationen, Illusionen \\
\hline - vermehrter Harndrang oder Blasenentlee- & - Gedächtnisstörung & - psychotische Symptomatik \\
$\begin{array}{l}\text { rungsstörung } \\
\text { - Kälteintoleranz }\end{array}$ & & - gestörte Impulskontrolle \\
\hline - Kreislaufdysregulation & & - Sexualfunktionsstörungen (Libido- \\
- erektile Dysfunktion & & steigerung, \\
\hline - REM-Schlaffunktionsstörungen & & Libidoverlust) \\
\hline - Insomnie & & \\
\hline - Tagesschläfrigkeit, Schlafattacken & & \\
\hline - Parästhesien, Schmerzen & & \\
\hline - Hyposmie, Anosmie & & \\
\hline
\end{tabular}




\section{Diagnose}

Die Bradykinese kann alle Körperabschnitte betreffen. Blickdiagnostisch stechen Haltung und Gang von Parkinsonerkrankten ins Auge. Die Muskelsteifigkeit manifestiert sich meist als erstes im Bereich des Nackens, der Schultern oder der Oberarme. Daraus resultieren einerseits Schmerzen, andererseits eine nach vorn gebeugte Haltung sowie eine prominente Schulter-Nacken-Partie („Stiernacken“). Beim Gang fehlt als Frühsymptom häufig das Mitschwingen der Arme, einoder beidseitig. Mit Fortschreiten der Erkrankung wird er kleinschrittig, schlurfend, unsicher. Häufig kann eine Starthemmung beobachtet werden. Zusätzlich fällt oft sehr früh eine abnehmende Mimik auf, die bis zur Amimie bzw. zum Maskengesicht führen kann. Die Lidschlagfolge verringert sich, häufig bleibt der Mund halboffen stehen.

\section{Klinische Untersuchung}

Nach der Inspektion von Mimik, Haltung und Gangverhalten erfolgt die Prüfung der Leitsymptome des Morbus Parkinson (siehe dazu auch die Infobox 1).

\section{- Tremor}

- Typ I: Ruhetremor („klassischer

Parkinsontremor“)

- tritt vor allem auf, wenn Erkrankte die Hände nicht gebrauchen, sondern diese z. B. im Schoß liegen

- ist durch kognitive Ablenkung (von 100 abwärts zählen) demaskierbar

- „Pillen drehende“ Bewegung zwischen Daumen und Zeigefinger

\section{INFOBOX 1}

Kriterien für die Diagnosestellung (Leitlinie der Deutschen Gesellschaft für Neurologie, DGN)

1. Feststellung der Akinesie/Bradykinese mit mindestens einem der folgenden Leitsymptome:
a) muskulärer Rigor
b) Ruhetremor
c) posturale Instabilität

2. Prüfung des Vorhandenseins unterstützender Diagnosekriterien:

a) einseitiger Beginn und persistierende Asymmetrie im Krankheitsverlauf

b) klassischer Ruhetremor

c) eindeutig positives Ansprechen auf L-Dopa

d) anhaltende L-Dopa-Ansprechbarkeit über mehr als 5 Jahre

e) Auftreten von L-Dopa-induzierten choreatischen Dyskinesien

f) langsame klinische Progression mit Krankheitsverlauf über mehr als 10 Jahre

3. Fehlen von Ausschlusskriterien für die klinische Diagnosestellung einer Parkinson Erkrankung
- häufig beim Gehen sichtbar, gleichzeitig mit fehlendem Mitschwingen der Arme

- typisch ist eine abnehmende Amplitude bei Bewegung, eine zunehmende Amplitude bei emotionaler Aufregung oder Anspannung

- Typ II: Ruhe- und Haltetremor

- Mischform

- Haltetremor tritt auf, wenn der Betroffene Kraft braucht, um Finger, Arm oder Bein in Position zu halten (z. B. Zittern beim Halten eines Wasserglases)

- Typ III: Reiner Halte- und Bewegungstremor

- Bewegungstremor tritt bei bestimmten Bewegungen auf, kann diese behindern (z. B. Klavierspielen oder Schreiben)

- Halte- und Bewegungstremor sind typische Kennzeichen des essenziellen Tremors, kommen aber auch beim Parkinson-Syndrom vor.

\section{- Rigor}

- langsame, passive Flexion/Extension der Ellbogen oder des Handgelenks mit Zahnrad-Phänomen (es ist keine gleichmäßige, sondern eine abgehackte, sakkadenartige Bewegung gegen einen Widerstand zu spüren)

\section{- Bradykinese}

- Schwierigkeiten beim Bewegungsbeginn und während der Durchführung von Bewegungen

- weniger und langsamere Bewegungen

- Prüfung durch Aufforderung, schnelle, wiederholte Bewegungen auszuführen: mit den Fingern trommeln, Diadochokineseprüfung (schnell aufeinander folgende Pro- und Supination der Hände)

- Posturale Instabilität (Störung der aufrechten Körperhaltung)

- nicht primär durch visuelle, vestibuläre, zerebelläre (unkontrollierte überschießende Bewegungen) oder propriozeptive Störungen (gestörte Koordination) erklärbar

- Prüfung beim stehenden Patienten mit angelegten Armen und geschlossenen Beinen: bei sanftem Stoß von hinten kommt es zur verlangsamten Ausgleichsbewegung mit Tendenz zum Fall und mehreren notwendigen Ausfallschritten

\section{Laboruntersuchung}

Zum Ausschluss anderer Ursachen der Symptome bzw. sekundärer Parkinson-Syndrome sind folgende Laborparameter aufschlussreich:

- Hämoglobin

- Leukozyten

- BSG

- Schilddrüsenwerte (TSH)

- Leberwerte (GGT, AP, GOT, GPT)

- Glukose

- Kreatinin 
- Bei Erkrankten<50 Jahren: Serumkupfer und Coeruloplasmin (Ferroxidase I) zum Ausschluss eines Morbus Wilson

\section{Neurologische Abklärung und Bildgebung}

Bei hausärztlicher Verdachtsdiagnose sollte der Patient zur Sicherung der Diagnose sowie zum Ausschluss anderer Differenzialdiagnosen an eine Fachpraxis für Neurologie überwiesen werden. Folgende bildgebende Maßnahmen sind möglich:

- CT/MRT

Zum Ausschluss sekundärer Ursachen des Parkinson-Syndroms (z. B. vaskuläres Parkinson-Syndrom) sollte eine zerebrale Bildgebung - CT oder MRT erfolgen.

- Transkranielle Sonografie

Zur Frühdiagnostik des Parkinson-Syndroms sowie zur Unterscheidung zwischen idiopathischem, atypischem und sekundärem Parkinson-Syndrom kann eine transkranielle Sonografie der Regio Substantia nigra mit nachweisbarer Hyperechogenität dienen. Da die Untersuchung jedoch viel Erfahrung verlangt, sollte sie mit anderen Bildgebungen verbunden werden.

- DAT-SPECT

Zur Unterscheidung zwischen idiopathischem Parkinson-Syndrom und essenziellem Tremor bei unklarer Symptomatik kann die Messung präsynaptischer Dopamin-Transporter mittels SPECT (DATSPECT) helfen. Eine reduzierte Verfügbarkeit der Transporter zeigt eine nigrostriatale Degeneration an. Bei rein arzneimittelinduziertem ParkinsonSyndrom ist diese Untersuchung unauffällig, bei vaskulärem Parkinson-Syndrom kann sie unauffällig sein.

- FDG-PET

Zur Abgrenzung atypischer neurodegenerativer Parkinson-Syndrome kann der Einsatz von Fluordesoxyglucose Positronen-Emissions-Tomografie (FDG-PET) indiziert werden. Diese Untersuchung bildet den regionalen zerebralen Glukosestoffwechsel ab, der eng an die neuronale Aktivität gekoppelt ist. Differenzialdiagnostisch lassen sich damit potenziell krankheitsspezifische Befundmuster für die jeweiligen neurodegenerativen Parkinson-Syndrome erkennen.

\section{Merke}

Eine frühe genaue und spezifische Abklärung kann rasch zur richtigen Therapie führen und den Betroffenen und Angehörigen viel Zeit der Unsicherheit und eingeschränkten Lebensqualität ersparen.

\section{Medikamentös induzierte Parkinson-Symptomatik}

\section{Diagnostische Überlegungen}

\section{Ausschlusskriterien}

Ausschlusskriterien für die klinische Diagnose eines Morbus Parkinson sind:

- Behandlung mit Neuroleptika oder Exposition gegenüber anderen Morbus Parkinson auslösenden Medikamenten oder Toxinen in zeitlichem Zusammenhang mit Erstmanifestation der ParkinsonSymptome

- Nachweis struktureller Basalganglienveränderungen, frontaler Tumoren oder Hydrocephalus communicans in der zerebralen Bildgebung

- wiederholte zerebrale ischämische Insulte, die mit einer stufenweisen Verschlechterung der ParkinsonSymptomatik assoziiert waren

- rezidivierende Schädel-Hirn-Traumen in der Vorgeschichte

- diagnostisch gesicherte Enzephalitis in der Vorgeschichte

- Remissionen über längere Perioden (DGN Leitlinie)

\section{Warnsymptome}

Warnsymptome, die auf ein atypisches Parkinson-Syndrom bzw. andere Symptomursachen hinweisen können, sind:

- Nicht-Ansprechen auf hohe Dosen von L-DOPA (> $1000 \mathrm{mg} / \mathrm{d}$ )

- frühzeitige, im Verlauf auftretende schwere Störungen des autonomen Nervensystems (orthostatische Hypotension, Synkopen, Harninkontinenz, Impotenz...)

- frühe häufige Stürze, insbesondere nach hinten

- innerhalb des ersten Jahres auftretende Demenz

- innerhalb des ersten Jahres auftretende Halluzinationen

\section{FALLBEISPIEL 2}

Medikamentös induzierte Parkinson-Symptomatik Eine 75-jährige Patientin kommt mit ihrer Tochter in die Ordination. Seit Jahren leide die Mutter unter Depressionen und Ängsten, sie stehe unter laufender Neuroleptikatherapie. In den letzten Monaten verschlechtere sich der Zustand zunehmend. Sie leide unter einer zunehmenden Gang- und Bewegungsstörung, verliere ihre Mimik, werde zunehmend hilfsbedürftiger. Außerdem falle ein vermehrtes Zittern auf, wechselnd im Bereich der Arme, des Kopfes, zwischendurch sei der ganze Körper betroffen. Im Rahmen eines stationären Aufenthaltes auf einer psychiatrischen Abteilung sei ein dopaminerges Medikament verordnet worden, seither habe sich keine Besserung gezeigt. Die Tochter nehme weiterhin eine Verschlechterung wahr. 
- stark fluktuierende Vigilanz und Müdigkeit

- Somnolenzphasen

- ausgeprägte Dysarthrie

- ausgeprägte Dysphagie

- ausgeprägter Antekollis

- zerebelläre Zeichen

- positiver Babinski-Reflex

- Apraxie und/oder Aphasie

- supranukleäre vertikale Blickparese (DGN Leitlinie)

\section{Relevante Substanzen}

Nach der populationsbasierten „Bruneck-Studie“ in einer Population $>50$ Jahre sind ca. $20 \%$ der diagnostizierten Parkinson-Syndrome auf eine medikamentöse Nebenwirkung zurückzuführen [4]. Meist lassen sich die Symptome erklären durch Medikamente mit antagonistischen Eigenschaften an striatalen Dopamin-D2Rezeptoren. Die wichtigsten Vertreter sind klassische Neuroleptika, atypische Neuroleptika, dopaminantagonistische Antiemetika und Kalziumantagonisten ( Tab.4).

Die Dauer der Neuroleptika-Einnahme scheint für das Risiko des Auftretens und die Schwere des medikamentös induzierten Parkinson-Syndroms keine Rolle zu spielen [6]. Nach Absetzen des ursächlichen Medikaments ist das medikamentös induzierte Parkinson-Syndrom reversibel, die Symptome können aber Wochen bis Monate anhalten.

\section{Klinische Diagnosekriterien}

Die klinischen Diagnosekriterien sind Parkinson-Symptome, die anamnestisch erst nach Beginn der Einnahme des verdächtigen Medikaments auftraten.

- Hinweisend ist ein bilateraler Beginn mit im Vordergrund stehender allgemeiner Bradykinese und Rigor, die posturale Stabilität ist häufig erhalten.

- Bei den meisten Erkrankten besteht nur eine leichte oder gar keine Gangstörung.

- Tab.4 Medikamente und Wirkstoffgruppen, die Auslöser für ein medikamentös induziertes Parkinson-Syndrom sein können [5].

\begin{tabular}{|c|c|}
\hline Häufige Auslöser & Seltene Auslöser \\
\hline $\begin{array}{l}\text { - typische Neuroleptika: } \\
\text { Phenothiazine, Butyrophenone, } \\
\text { Diphenylbutylpiperidine, } \\
\text { Benzamide } \\
\text { - atypische Neuroleptika: } \\
\text { Risperidon, Olanzapin, Aripi- } \\
\text { prazol } \\
\text { - Dopamin-Entspeicherer: } \\
\text { Tetrabenazin } \\
\text { - Antiemetika: } \\
\text { Metoclopramid } \\
\text { - Kalziumantagonisten: } \\
\text { Verapamil, Flunarazin }\end{array}$ & $\begin{array}{l}\text { - atypische Neuroleptika: } \\
\text { Clozapin, Quetiapin } \\
\text { - Stimmungsstabilisierer: } \\
\text { Lithium } \\
\text { - Antidepressiva: } \\
\text { SSRI } \\
\text { - Antiepileptika: } \\
\text { Valproinsäure, Phenytoin }\end{array}$ \\
\hline
\end{tabular}

- Ein klassischer Ruhetremor kann vorkommen, persistiert dann aber auch häufig als Aktionstremor.

- Viele Betroffene weisen assoziierte Bewegungsstörungen auf, wie unkontrollierte Bewegungen oder auffallende motorische Unruhe.

Die Klinik alleine lässt hier jedoch meist keine sichere Unterscheidung von einem idiopathischen ParkinsonSyndrom zu. In diesem Fall sollte ein Dopamintransporter-SPECT durchgeführt werden, welches beim medikamentös induzierten Parkinson normal ist-es sei denn, es liegt eine Überschneidung mit dem idiopathischen Parkinson-Syndrom vor, was bei älteren Erkrankten durchaus vorkommt.

\section{Merke}

Bei Nicht-Ansprechen der motorischen Symptome auf dopaminerge Medikamente in ausreichender Dosis muss die Erkrankungsätiologie überdacht werden.

\section{Angst bei früher Parkinson- Symptomatik}

\section{Psychische Komorbiditäten}

Depression, Angst

Rund $40 \%$ aller Parkinson-Erkrankten leiden an Depression, und ebenso viele der Betroffenen entwickeln im Laufe der Erkrankung eine Demenz [7, 8]. In der Literatur meist untergeordnet, jedoch in ähnlich hoher Prävalenz treten Angstsymptome sowie Angststörungen auf, insbesondere generalisierte Angststörung, Panik oder soziale Phobie [9]. Häufig zeigen sich emotionale und kognitive Beeinträchtigungen als Frühsymptom. Sowohl Depression als auch Angst haben erhebliche Auswirkungen auf den Verlauf der Erkrankung sowie auf die Lebensqualität [10]. Insbesondere die im Rahmen der Parkinson-Erkrankung beschriebenen motorischen Symptome können durch psychischen Stress verstärkt werden [11].

Pathophysiologisch sind Depression und Angst bei der Parkinson-Krankheit am ehesten als primäre Konsequenz degenerativer Veränderungen in katecholaminergen Neurotransmitter-Systemen und frontokortikalen Dysfunktionen zu sehen [12]. Angst entsteht häufig auch durch die zunehmenden motorischen Defizite mit Gangunsicherheit, Fallneigung und zunehmender Immobilität.

Merke

Angst kann aber auch als Therapienebenwirkung auftreten, beispielsweise bei einer L-Dopa-Therapie. 
Demenz, Schlafstörungen

Bei der Demenz sind zu unterscheiden

- die Parkinson-Demenz, die sich im Spätstadium entwickelt und ebenfalls Folge der neurodegenerativen Veränderungen ist, und

- die Lewy-Körper-Demenz, die in der Regel mit kognitiven Symptomen beginnt und motorische Parkinson-Symptome nach sich zieht [13].

Es kommt zu verringertem oder fehlendem Antrieb, abgeflachten Emotionen sowie zu einem generell niedrigeren Aktivitätsniveau.

Ein sehr häufiges Symptom stellen zudem Schlafstörungen dar, bis zu $60 \%$ der Parkinson-Erkrankten sind davon betroffen. Sie können Teil des Krankheitsbildes, aber auch Folge der Behandlung sein, treten in Form von Ein- und Durchschlafstörungen, Störungen des REM-Schlafes mit vermehrt physischer Aktivität während des Träumens sowie durch Tagesmüdigkeit und -schläfrigkeit auf. Natürlich zeigt sich hier ein Zusammenhang mit Dysthymie sowie Abnahme der Lebensqualität.

\section{Psychosoziale Auswirkungen}

Neben den beschriebenen Komorbiditäten muss die psychosoziale Problematik diskutiert werden, die Parkinson-Erkrankte im Allgemeinen in der sozialen Interaktion betrifft. Die reduzierte Mimik und die dadurch fehlende emotionale Reaktion im Gesicht hinterlässt bei Gesprächspartnern den Eindruck einer geistigen Einschränkung. Verstärkt wird dies durch die Veränderungen von Sprechen und Sprache. Die Fehleinschätzung durch das Gegenüber bedeutet für die Betroffenen eine oft beträchtliche soziale Benachteiligung.

Die Belastung durch die zunehmende körperliche Einschränkung ist ebenfalls groß, ebenso das Bewusstsein der stetigen Verschlechterung bei den Betroffenen sowie deren Angehörigen. Hier ist häufig die Angst vor Verlust von Autonomie, Mobilität sowie vor zunehmender Hilflosigkeit und Pflegebedürftigkeit groß. Hinzu kommt ein Schamgefühl aufgrund der zunehmenden Ungeschicklichkeit sowie der Veränderung von Mimik, Stimme, Beweglichkeit und Körperhaltung. Oft bewirkt dies den sozialen Rückzug, der in der Folge Depression und Ängste fördert. Nicht zuletzt sind partnerschaftliche Konflikte häufig, einerseits aufgrund des veränderten emotionalen sowie kognitiven Verhaltens, andererseits aufgrund des durch die Erkrankung und die Medikamente veränderten Sexualverhaltens. Psychosoziale Unterstützung ist oft von entscheidender Bedeutung für Betroffene und ihre Angehörigen.
FALLBEISPIEL 3

\section{Angst bei früher Parkinson-Symptomatik}

Ein 63-jähriger Patient wird in Ihrer Praxis vorstellig und berichtet von einem stattgehabten Kollaps während einer Reise. Er beschreibt, im Rahmen dieses Vorfalls große Angst verspürt und am ganzen Körper gezittert zu haben. Er habe dies nicht regulieren können. Nach 15 Minuten ruhigem Liegen sei die Symptomatik besser geworden. Seither falle ihm jedoch ein immer wieder kehrendes Zittern seiner linken Hand auf, was ihn sehr störe. Auf Nachfrage trete das Zittern eher auf, wenn die Hand nichts zu tun habe, Golfspielen funktioniere beispielsweise tadellos.

Der Patient zeigt Ihnen seine zitternde Hand, kann den Tremor aber mit Konzentration selbst abstellen. Sie untersuchen den Patienten, stellen keinen Rigor fest, die Mimik ist unauffällig. Beim Gehtest fällt Ihnen jedoch auf, dass der linke Arm weniger mitschwingt als der rechte. Der Patient scheint einen ausgeprägten Nackenhartspann zu haben, sein Kopf ist nach vorne geneigt. Schmerzen habe er keine, aber es falle ihm auf, dass seine Haltung schlechter werde. Auf die Frage, was er denn selbst glaube, was ihm fehle, antwortet der Patient: „Ich habe Angst, dass ich Parkinson bekomme. Mein Vater hatte das, es war schrecklich“.

\section{Merke}

Eine neurodegenerative Erkrankung wie das Parkinson-Syndrom hat umfassende biopsychosoziale Auswirkungen auf das Leben der Betroffenen und deren Angehörigen und muss dementsprechend mit verschiedensten Therapieansätzen behandelt werden.

\section{Medikamentöse Therapie}

Die medikamentöse Therapie des idiopathischen Parkinson-Syndroms erfolgt in erster Linie symptomatisch. Eine früher angenommene krankheitsmodulierende Wirkung einiger Substanzklassen ist mittlerweile umstritten. Der Therapiebeginn erfolgt je nach Leidensdruck des Betroffenen; Vorteile eines früheren oder späteren Therapiebeginns konnten in Studien nicht gezeigt werden. Individuell ist auch die Auswahl des Präparats, das auf Alter, Komorbiditäten und psychosoziale Problematik abgestimmt werden sollte. Schließlich werden nach Beginn der Therapie Wirkung und Nebenwirkungen evaluiert, gegebenenfalls wird die Dosierung angepasst bzw. auf ein anderes Präparat umgestellt.

\section{Levodopa}

In Kombination mit peripher wirkenden Decarboxylasehemmern ist Levodopa die effektivste Therapie, da sie den der Krankheit zugrunde liegenden Dopaminmangel direkt ausgleicht. L-Dopa ist eine Vorläufersubstanz von Dopamin, die durch den Magen-Darm-Trakt aufgenommen, im Gehirn zu Dopamin umgewandelt 
wird. Die Decarboxylasehemmer (Benserazid oder Carbidopa) verhindern eine vorzeitige Umwandlung von LDopa in Körpergeweben, in denen es nicht benötigt wird. Bei älteren Patienten/-innen wird diese Kombination initial eingesetzt, bei unzureichendem Effekt anderer Wirkstoffe auch bei jüngeren. Hier galt bislang eine therapeutische Entscheidungsgrenze ab dem 70. Lebensjahr, wobei diese angesichts einer mittleren Lebenserwartung von derzeit 81 Jahren relativ zu sehen ist (siehe dazu auch die Infobox 2).

\section{Dosierung, Dauer}

Man beginnt üblicherweise mit einer niedrigen Initialdosis eines nicht retardierten Präparates (z.B. $3 \times$ $50 \mathrm{mg}$ tgl. in Abständen von ca. 5 Stunden) und erhöht dann schrittweise über Tage bis Wochen, je nach Ansprechen und Nebenwirkungen. Eine längere Behandlungsdauer geht einher mit einer erhöhten Wahrscheinlichkeit für Spätkomplikationen in Form von Wirkungsfluktuationen und Dyskinesien; entsprechende Phänomene findet man bei $40 \%$ der Patienten/-innen nach 5 Jahren L-Dopa-Behandlung. In frühen Erkrankungsstadien sollte daher anderen Präparaten der Vorzug gegeben werden. Insbesondere zur Therapie nächtlicher motorischer Symptome bleibt Levodopa in retardierter Form aber auch initial Therapie der Wahl.

Mit der Zeit verringert sich die Wirkungsdauer jeder Dosis. Depotpräparate können verwendet werden, erfordern jedoch höhere Dosen. Eine weitere Anwendungsform stellt die kontinuierliche intestinale Applikation von Duodopa (Kombination aus L-Dopa und Carbidopa in Gelform) mittels PEG-Sonde dar.

\section{INFOBOX 2}

\section{Indikation von Levodopa bei Parkinson-Erkrankten}

\section{$<70$ Jahren}

- unzureichende Wirkung von Dopaminagonisten

- Unverträglichkeit von Dopaminagonisten:

- Tagesmüdigkeit

- Ödeme

- Halluzinationen

- Orthostase

- Impulskontrollstörungen

- Multimorbidität, insbesondere bei

- metabolischem Syndrom

- kardiovaskulären Erkrankungen

- zerebrovaskulären Erkrankungen

- gewünschter rascher Behandlungseffekt

- therapeutische Diagnostik

\section{Unerwünschte Wirkungen}

Zu den unerwünschten Wirkungen des Levodopa zählen

- Dyskinesien,

- On-Off-Phänomen,

- Übelkeit,

- Schwindel,

- orthostatische Hypotension.

Neben Dyskinesien kommt es bei 25-50\% der Behandelten nach 5 Jahren auch bei niedriger Dosierung zu motorischen Fluktuationen, bei Jüngeren treten diese sogar in >90\% der Fälle nach 5 Jahren auf [14].

Betroffene erleben extreme Schwankungen zwischen guter Beweglichkeit („On“) und Unbeweglichkeit („Off“) - das sogenannte On-Off-Phänomen. Es kann sowohl zu schnellerem Abklingen der Levodopa-Wirkung mit tagesschwankender Beweglichkeit („End-ofDose-Akinesie“) kommen als auch zum plötzlichen Aussetzen der Wirkung („Sudden off“). Treten die beschriebenen Nebenwirkungen unter reiner LevodopaTherapie auf, kann versucht werden, die Therapie mit einem COMT-Hemmer, MAO B-Hemmern oder Dopaminagonisten zu kombinieren. Auch eine häufigere Gabe von Levodopa in kleineren Dosen kann hilfreich sein.

Häufig beklagte Nebenwirkungen sind außerdem Übelkeit und Schwindel, wobei der Schwindel auch krankheitsassoziiert sein kann. Zusätzlich sturzgefährdend kann sich die als Nebenwirkung beschriebene orthostatische Hypotension auswirken. Da diese vor allem zu Beginn der Therapie auftritt, sollten die Patienten/-innen explizit darauf hingewiesen werden. Die Dosierungen von Levodopa sollten generell langsam erhöht werden, dabei sollte man über Wochen auf eine wirksame Dosis steigern.

\section{Merke \\ Einige Symptome der Parkinson-Erkrankung wie Schlafstörungen, Halluzinationen, orthostatische Dysregulation, Bewegungsstörungen usw. können durch die medikamentöse Therapie verstärkt bzw. erst ausgelöst werden.}

\section{Dopamin-Agonisten}

Dopamin-Agonisten sind dem körpereigenen Dopamin chemisch sehr ähnlich, haben jedoch eine schwächere Wirksamkeit als L-Dopa. Unterschieden werden Ergolin-basierte und nicht-Ergolin-basierte Dopamin-Agonisten. Da Ergolin-basierte Dopamin-Agonisten zu fibrotischen Veränderungen an Herz, Retroperitoneum und Lunge führen können, sollten diese nur in Ausnahmefällen verschrieben und nicht-Ergolin-basierten Medikamenten der Vorzug gegeben werden. 
Dopamin-Agonisten werden insbesondere bei jüngeren Erkrankten vor dem 70. Lebensjahr zu Beginn der Behandlung als Monotherapie verabreicht. Außerdem kommen sie in Kombination mit Levodopa zum Einsatz, um motorische Fluktuationen oder zunehmende „OffPhasen“ bei Erkrankten mit fortgeschrittenem idiopathischen Parkinson-Syndrom zu reduzieren. Begonnen wird mit der niedrigstmöglichen Dosierung, die noch eine Kontrolle der Symptome ermöglicht; wenn nötig, erfolgt eine Steigerung in wöchentlichen Intervallen. Einen Dopamin-D2-Rezeptor-Agonisten stellt Apomorphin dar, das als kontinuierliche subkutane Infusion mittels Pumpe oder als Pen zum Einsatz kommt.

\section{Nebenwirkungen}

Aufgrund des Nebenwirkungsprofils mit gehäuften kognitiven Störungen sollten Dopaminagonisten bei Betroffenen mit kognitiver Leistungseinschränkung, Demenz und/oder psychotischem Erleben nicht eingesetzt werden. Neben Beinödemen, Übelkeit und Schwindel werden auch häufig Tagesmüdigkeit bis hin zu Einschlafattacken beschrieben. Dies ist jedoch präparatabhängig: unter Piribedil wird weniger Tagesmüdigkeit berichtet, unter Rotigotin eine Verbesserung des Nachtschlafs, und unter Pramipexol eine antidepressive Wirkung.

Vor allem psychosozial belastend sind Störungen der Impulskontrolle, die unter allen dopaminergen Medikamenten auftreten können. Sie äußern sich vor allem in Form von Spielsucht, Libidosteigerung/Hypersexualität, Kauf- oder Esszwang, aber auch als emotionale Impulskontrollstörung.

\section{MAO-Hemmer Typ B}

MAO-B-Hemmer hemmen den Dopaminabbau im Gehirn. Sie können initial als Monotherapie oder in Kombination mit Levodopa eingesetzt werden. Auch eine Kombination mit COMT-Hemmern kann eine Option sein. Zur Kombinationstherapie eignet sich in erster Linie Rasigilin; Selegilin sollte eher als Monotherapie angewendet werden. Bei älteren Patienten/-innen wird Selegilin nicht empfohlen, und zwar aufgrund der möglichen Bildung von Amphetamin-ähnlichen Metaboliten und damit zusammenhängender Orthostase, Refluxsymptomatik sowie Schlafstörungen.

Cave

Wichtige Komplikation: Bei Kombination mit anderen serotonergen Medikamenten kann möglicherweise ein Serotonin-Syndrom auftreten.

\section{COMT-Hemmer}

Catechol-O-Methyl-Transferase-Hemmer vermindern ebenfalls den Dopaminabbau im Gehirn. Sie können somit die Bioverfügbarkeit von Levodopa erhöhen und deren Wirkungsdauer verlängern. Anders als MAO-BHemmer sind sie als Monotherapie nicht wirksam. Sie können bei Erkrankten mit Fluktuationen als Kombination mit Levodopa angezeigt sein und durch die Verlängerung der Wirkungsdauer eine Dosiserhöhung von Levodopa hinauszögern. Auch eine Kombination mit MAO-B-Hemmern ist eine mögliche Option. Entacapon sollte dem Tolcapon wegen des Nebenwirkungsprofils eher vorgezogen werden. Neben einer Verstärkung der Levodopa-Wirkung kann es auch zu verstärkten Nebenwirkungen kommen, zu Diarrhoe und Orangefärbung des Harns.

\section{NMDA Rezeptor-Antagonisten}

Der Dopaminmangel bewirkt im striatalen Regelkreis eine relative glutamaterge Überaktivität. Ein anderer medikamentöser Ansatzpunkt ist es daher, den Neurotransmitter Glutamat über eine Antagonisierung des N-Methyl-D-Aspartat (NMDA)-Subtyps des striatalen Glutamatrezeptors zu bremsen. Dadurch erfolgt ein Ausgleich der dopaminergen Hemmung bzw. der glutamatergen Stimulation cholinerger Neurone, was zur Verbesserung des Tremors und auch der Dyskinesien führt. Auch eine gute Wirksamkeit bei Bewegungsstarre ist beschrieben.

NMDA Rezeptor-Antagonisten können in der frühen Parkinson-Therapie als Mittel zweiter Wahl eingesetzt werden; auch bei medikamentös induziertem Parkinson-Syndrom zeigen sie durch den Ausgleich der oben beschriebenen Botenstoffe häufig Erfolg.

\section{Weitere symptombezogene medikamentöse Optionen}

- Tremorsymptomatik

Nicht als Mittel erster Wahl, jedoch zur alternativen symptomatischen Therapie des Tremors können Betablocker oder Anticholinergika versucht werden (letztere nicht bei Älteren bzw. Erkrankten mit Demenz!).

- Sialorrhoe (Speichelfluss aus dem Mund) Bei der gesellschaftlich sehr unangenehmen Sialorrhoe spielen eine verminderte Schluckfähigkeit und/ oder eine erhöhte Speichelproduktion eine Rolle. Eine ausreichende Dopaminersatz-Therapie ist Grundlage der Therapie. Reicht diese nicht aus, sind lokale Injektionen von Botulinumtoxin in die Speicheldrüsen (off-label) und anticholinerg wirksame Medikamente zu diskutieren. Eine sorgfältige RisikoNutzen-Abwägung ist dabei angezeigt.

- Psychotische Symptomatik In erster Linie kommen Infekte, Dehydratation und die Polypharmazie mit den zentral wirksamen AntiParkinson-Medikamenten als Auslöser in Frage. Insofern sind die Infektbehandlung und die Flüssigkeitssubstitution relevant. Häufig sind die Gabe von Antipsychotika der 2. Generation sowie eine Dosis- 
reduktion der laufenden Anti-Parkinson-Medikation jedoch unerlässlich. Niedrig dosiertes Quetiapin (z. B. Beginn mit 12,5 mg abends und allmähliche Steigerung) kommt hier in Frage.

\section{Nichtmedikamentöse Therapie Tiefe Hirnstimulation}

Bei Patienten/-innen mit medikamentös nicht behandelbaren motorischen Fluktuationen und Dyskinesien oder medikamentös nicht kontrollierbarem Tremor kann an eine tiefe Hirnstimulation gedacht werden. Dabei erfolgt eine elektrische Stimulation des Nucleus subthalamicus, des Thalamus oder Globus pallidus mittels neurochirurgisch (unter laufender Bilddiagnostik) eingebrachter Elektroden. Diese Methode zeigte in mehreren Studien eine gute Wirkung auf die Morbus Parkinson Leitsymptome Rigidität, Bradykinese und Tremor. Sie verringert Levodopa-induzierte Hyperkinesen und Off-Zeiträume signifikant. Neben üblichen neurochirurgischen Komplikationen können Nebenwirkungen auftreten durch die Stimulation an sich: Apathie und Depression, Verstärkung von Dyskinesie und Gehstörungen, Parästhesien, Einschränkungen der Wortflüssigkeit und des Arbeitsgedächtnisses. Meist sind diese Nebenwirkungen jedoch reversibel.

Voraussetzungen für eine tiefe Hirnstimulation sind:

- Leitsymptome sprechen auf Levodopa an, Tremor muss nicht zwingend ansprechen

- keine Frühsymptome einer Demenz

- keine signifikante psychische oder somatische Komorbidität

- keine neurochirurgischen Kontraindikationen

- individuelle Abwägung der Risiken des operativen Eingriffs gegen den Gewinn durch die Therapie

\section{Bewegungstherapie}

Neben der medikamentösen Behandlung stellt die Bewegungstherapie eine der tragenden Säulen der Therapie des Morbus Parkinson dar. Es kommt bei den Betroffenen ja nicht nur zu den motorischen Kardinalsymptomen, sondern auch zu einer Beeinträchtigung der intra- und intermuskulären Koordination. Dies trägt maßgeblich dazu bei, dass die Schwierigkeiten in der Durchführung komplexer motorischer Abläufe zunehmen (z. B. das Sich-Erheben aus einem Stuhl).

\section{Merke}

Bewegung wirkt sich positiv auf das Gehirn aus, indem sie die Bildung von Hirnzellen stimuliert und die Ausschüttung von Dopamin anregt.

Studien zeigen, dass Personen, die an unterschiedlichen Bewegungsübungen teilnahmen, beweglicher waren, schneller gehen und ihr Gleichgewicht besser halten konnten als Personen, die nicht an den Übungen teilnahmen. Auch eine geringere Sturzrate bei regelmäßiger Bewegung konnte festgestellt werden. Steigerung von Mobilität und Beweglichkeit im Alltag sowie eine Verbesserung der Koordination erleichtern die Bewältigung von Alltagsaktivitäten [15-17].

Erkrankte im Frühstadium sollten, sofern sie bereits regelmäßig Sport treiben, dazu angeregt werden, Art und Umfang zu erhöhen. Für Menschen, die bislang keine Affinität zu sportlichen Aktivitäten hatten, wird der Beginn eines regelmäßigen Bewegungstrainings (moderates Ausdauer-, Kraft- als auch Koordinationstraining) empfohlen.

Bei stärkeren Einschränkungen kommt einer gezielten parkinsonspezifischen Physiotherapie eine wichtige Bedeutung zu. Besondere Schwerpunkte sollten dabei Gang- und Gleichgewichtstraining, Kraft- und Dehnübungen sowie Training von Bewegungsstrategien und Koordination sein. Ziel ist der Erhalt oder die Verbesserung von Gleichgewicht, aerober Kapazität, Bewegungsamplituden, Bewegungsinitiierung, Mobilität und Selbstständigkeit bei Aktivitäten des täglichen Lebens. Wesentliches Augenmerk sollte außerdem auf die Sturzprävention gelegt werden.

\section{Ergo-, Logo- und Psychotherapie}

- Ergotherapie

Ziel ist der Erhalt der Funktionsfähigkeit in Beruf und Zuhause, der autonomen Mobilität (z. B. Verwendung öffentlicher Verkehrsmittel) und der Möglichkeit für lebensförderliche Freizeitaktivitäten. In späteren Stadien stehen Aktivitäten des täglichen Lebens wie Essen, Trinken, Waschen, Kleiden und Toilettengang im Vordergrund.

- Logotherapie

Zielrichtungen sind die Verbesserung von Lautstärke und Verständlichkeit des Sprechens, und bei Schluckstörungen ein Schlucktraining.

- Psychotherapie Psychosoziale Unterstützung der Betroffenen und der Angehörigen ist in verschiedenen Phasen der emotionalen Überlastung, bei ausgeprägter Ambivalenz bzgl. Prognose und Lebensqualität und mangelnder Akzeptanz der Erkrankung sehr sinnvoll. Es gibt auch Hinweise für den Erfolg von Kunst- Musikoder Tanztherapie.

\section{Notfälle}

\section{Akinetische Krise}

In späten Stadien kann es zum plötzlich eintretenden Zustand einer völligen Bewegungsunfähigkeit kommen, zur der man eventuell als Arzt für Allgemeinmedizin gerufen wird. Meist liegt ein ausgeprägter Rigor vor, durch den die Erkrankten weder sprechen noch 
schlucken können. Durch die Schluckstörung fehlt die Medikation, besteht Aspirationsgefahr und findet keine Flüssigkeitsaufnahme statt. Es handelt sich um einen lebensbedrohlichen Notfall, der in der Regel stationär behandelt werden muss.

Eingeschränkte Atmung, Hyperthermie mit ausgeprägtem Schwitzen, Pulsanstieg, Aspiration, Thromboembolien sind die zu beherrschenden Risiken. Auslöser einer solchen akinetischen Krise sind meist fieberhafte Infektionskrankheiten, Unterbrechung der ParkinsonMedikation oder die hochdosierte Gabe von Neuroleptika. Medikamente der Wahl sind Amantadin intravenös, Apomorphin subkutan oder L-Dopamin über eine Magensonde.

\section{Stürze}

Schon banale Ablenkungen beim Gehen (z. B. Gedränge, Hinweise auf ein fernes Objekt oder Unterhaltung) können einen Sturz provozieren, ursächlich sind die Verlangsamung motorischer Reflexe und die Schwierigkeit, verschiedene Aufgaben gleichzeitig auszuführen. Das Gleiche gilt auch für kleine Hindernisse bzw. Unebenheiten des Bodens. Das hat für prophylaktische Maßnahmen in der Wohnung Bedeutung, aber auch für das Anraten von Hilfsmitteln beim Gehen (Wanderstöcke).

\section{Psychose}

Psychotische Symptome beziehen sich auf Wahnvorstellungen bei fehlender Einsicht. Risikofaktor ist eine demenzielle Begleiterkrankung oder eine Depression, Auslöser oft die medikamentöse anticholinerge Therapie. Metabolische Probleme oder Infekte sollten zunächst weitgehend ausgeschlossen werden, die anticholinerge Therapie sollte man anpassen. Atypische Neuroleptika mit geringer extrapyramidalmotorischer Wirkung sind eventuell zu erwägen [18].

\section{Suizidalität}

Parkinson-Kranke haben ein 1,87-fach erhöhtes Suizidrisiko [19]. Ein Augenmerk der betreuenden Ärzte/Ärztinnen auf die Krankheitsbewältigung ist daher von Bedeutung. Notwendig sind gute Informationen über die vermuteten Ursachen, über die therapeutischen Möglichkeiten, den wahrscheinlichen Verlauf, die Chancen durch Medikamente und Selbsthilfemaßnahmen sowie über den Kontakt bei Krisen.

\section{Merke}

Depression und Angststörungen betreffen im Laufe der Erkrankung fast die Hälfte der ParkinsonErkrankten. Auch Suizidalität sollte daher von den behandelnden Ärzten und Ärztinnen bedacht und aktiv angesprochen werden.

\section{FALLBEISPIEL 4}

\section{Drohende Suizidalität}

Die Gattin eines 70-jährigen Patienten wird vorstellig. Ihr Mann habe vor 3 Jahren die Diagnose Morbus Parkinson erhalten. Trotz Medikation verschlechtere sich sein Zustand zunehmend, Treppensteigen funktioniere kaum noch, insgesamt werde das Gehen immer schwieriger. Er sei verzweifelt, spreche immer wieder davon, sich das Leben nehmen zu wollen. Sie wisse nicht mehr, wie sie ihn adäquat unterstützen könne.

\section{Freezing und orthostatische Dysregulation}

Unter dem Begriff „Freezing“ wird die plötzliche Blockade von Bewegungen bzw. Erstarren verstanden. Etwa ab dem 5. Jahr der Manifestierung einer Parkinson-Erkrankung tritt dieses Phänomen bei ungefähr der Hälfte der Erkrankten auf. Es kann sehr kurz oder bis zu 30 Sekunden dauern und kann bei Überqueren einer Straße oder Verlassen eines Verkehrsmittels zur großen Gefahr werden. Verschiedene Arten von Freezing werden beschrieben und stellen auch für Spezialisten eine große Herausforderung dar.

Von der American Parkinson Disease Assoziation gibt es Empfehlungen, um Freezing aufzulösen, von Richtungswechsel bis zu verschiedenen Ablenkungsversu-

\section{FALLBEISPIEL 5}

Freezing und orthostatische Dysregulation

Ein Ihnen langjährig bekannter Parkinson-Patient wird regelmäßig bei Ihnen zu Routinekontrollen vorstellig. Regelmäßig kommt es während der Konsultationen zu sichtbarer Verlangsamung („Freezing“) des Patienten mit plötzlicher Sprach- und Bewegungsarmut. Bei der heutigen Konsultation berichtet der Patient, dass ihm in letzter Zeit öfter schwarz vor Augen werde, er verliere häufig kurzzeitig das Bewusstsein.

\section{PRAXIS}

\section{Empfehlungen bei Freezing}

- eine alternative Bewegung zur Ablenkung mit den Armen durchführen (Arm zum Himmel heben, den Kopf berühren etc.)

- die Bewegungsrichtung ändern

- einen Punkt am Boden fixieren und diesen ansteuern

- ein Lied summen und zum Takt marschieren

- bis 3 zählen, dann gehen

- Gewichtsverlagerung von einer Seite zur anderen

- ein paar Schritte auf der Stelle, dann vorwärts gehen 
chen (siehe Box). Natürlich ist auch die medikamentöse Therapie zu überdenken.

\section{Internistische Aspekte des Parkinson-Syndroms}

\section{Autonome Dysfunktion}

\section{Symptomatik}

Vor allem atypische Parkinson-Syndrome gehen häufig einher mit einer frühen Störung des vegetativen (= autonomen) Nervensystems. Diese autonome Dysregulation kann sich mit einer schweren Funktionsstörung im Sinne der orthostatischen Hypotonie $(\mathrm{OH})$ oder mit vasovagalen Synkopen (VVS) manifestieren. Ferner sind auch das posturale orthostatische Tachykardie-Syndrom (POTS), das Karotissinus-Syndrom und eine in liegender Position häufig in der Nacht auftretende arterielle Hypertonie zu nennen. Die möglichen klinischen Symptome aller einer orthostatischen Dysfunktion zugrundeliegenden kardiovaskulären Störungen umfassen Benommenheit, Schwindel, Erschöpfung, Müdigkeit, Konzentrationsschwierigkeiten, VerschwommenSehen, Zittrigkeit, Blässe, Angst, Palpitationen, Frösteln oder Übelkeit [20].

Neben einigen weiteren Entitäten der atypischen Parkinson-Syndrome (wie beispielsweise die Lewy-BodyDemenz, die progressive supranukleäre Parese oder die kortikobasale Degeneration, die nicht typischerweise mit einer autonomen Dysregulation einhergehen) spielen hier in erster Linie atypische ParkinsonSyndrome eine entscheidende Rolle. Sehr häufig präsentiert sich diese mit dem Leitsymptom einer schweren orthostatischen Dysfunktion. Man unterscheidet 2 Formen der MSA in Abhängigkeit von der motorischen Begleitsymptomatik:

- die MSA-C (zerebelläre Form mit Atrophie in olivoponto-zerebellärer Lokalisation) und

- die MSA-P (Parkinson-Form mit striato-nigraler Degeneration).

Ein generalisiertes autonomes Versagen ohne ZNS-Beteiligung wird bezeichnet als isolierte autonome Insuffizienz, idiopathische orthostatische Hypotonie oder (nach den Erstbeschreibern) Bradbury-Egglestone-Syn- drom. Im Unterschied zur MSA fehlt hier eine präganglionäre oder zentrale Beteiligung, die sich je nach Lokalisation in motorischen Symptomen zerebellärer oder parkinsonoider Art äußert. Der MSA und der isolierten autonomen Insuffizienz gemeinsam ist hingegen, dass die autonome Störung neben dem kardiovaskulären Symptomkomplex häufig einhergeht mit

- gastrointestinalen Symptomen (Obstipation, verzögerte Magenentleerung, Dysphagie) oder

- urogenitalen Symptomen (Miktionsstörung, Inkontinenz, Nykturie, erektile Dysfunktion).

Die beschriebenen gastrointestinalen Symptome beruhen auf einer generellen gastrointestinalen Hypomobilität mit verzögerter Peristaltik. Häufig kommt es zur Dysfunktion des Pylorus sowie der lleozökalklappe. Bakterielle Dysbalance und „Leaky gut Syndrom“ werden als Folge diskutiert [21].

Weitere mögliche Manifestationen der autonomen Dysfunktion umfassen Atembeschwerden durch einen permanenten inspiratorischen Stridor, ein Schlafapnoe-Syndrom, eine herabgesetzte Empfindlichkeit auf hypoxische oder hyperkapnische Stimuli, eine Fehlfunktion der Schweiß- und Talgdrüsen mit An- oder Hyperhidrose und Seborrhoe, die Xerophthalmie oder eine durch sympathische Fehlinnervation der AlphaAdrenozeptoren im Bereich der Arteriolen vermittelte Vasokonstriktion, die zu einem Raynaud-Phänomen führen kann ( $\triangleright$ Tab.5).

\section{Therapeutische Optionen}

Entsprechend dem betroffenen dysautonomen Gebiet stehen nur symptomatische nichtmedikamentöse und medikamentöse Maßnahmen zur Verfügung.

- Bei der orthostatischen Dysregulation können beispielsweise physikalische Maßnahmen zur Unterstützung der Muskelpumpe oder das Tragen von Kompressionsstrümpfen zur Anwendung kommen.

- Als mögliche medikamentöse Therapieoptionen stehen zur Steigerung des Intravasalvolumens beispielsweise Fludrocortison (synthetisches Mineralokortikoid) oder zur verstärkten Vasokonstriktion Sympathomimetika wie Midodrin zur Verfügung.

- Zur Behandlung der genannten gastroenterologischen Manifestationen können Laxantien wie Ma-

\Tab. 5 Häufige Symptome im Rahmen der autonomen Dysfunktion.

\begin{tabular}{|l|l|l|}
\hline kardiovaskulär & gastrointestinal & urogenital \\
\hline - Schwindel & " gastrointestinale Hypo- & " Miktionsstörung \\
\hline - Müdigkeit & mobilität & - Inkontinenz \\
\hline - Konzentrationsstörung & " Dysfunktion Pylorus und Ileo- & " Nykturie \\
- Verschwommen-Sehen & zökalklappe & " erektile Dysfunktion \\
\hline - Zittrigkeit & " Übelkeit & \\
\hline - Palpitationen & & \\
\hline
\end{tabular}


crogol oder Prokinetika wie Domperidon eingesetzt werden. Obstipationsfördernde Medikamente wie Protonenpumpeninhibitoren sollten möglichst vermieden werden. Da jedoch die Prävalenz einer gastroösophagealen Refluxerkrankung mit $65 \%$ vier Jahre nach Diagnosestellung eines Parkinson-Syndroms äußerst hoch ist, gerät man hier oft in einen therapeutischen Konflikt [22].

- Desmopressin wird bei Nykturie angewandt.

- Anticholinergika wie Oxybutinin oder Trospium haben als Spasmolytika einen Stellenwert in der Behandlung von Harninkontinenz, können sich aber wiederum negativ auf die gastrointestinalen und auf demenzielle Symptome auswirken.

- Tamsulosin oder Doxazosin kommen bei Harnverhalt zur Anwendung, wobei Doxazosin als AlphaBlocker seinerseits eine orthostatische Intoleranz verstärken kann.

- Sildenafil oder Alprostadil werden gegen erektile Dysfunktion eingesetzt.

- Mittels Injektion von Botulinum-Toxin in die Stimmband-Adduktoren kann ein Stridor behandelt werden, und ein Schlafapnoe-Syndrom mittels nächtlicher CPAP-Maske.

\section{Merke}

Symptome einer autonomen Dysfunktion sind bei Parkinson-Syndromen häufig. Sind diese bereits in einem frühen Erkrankungsstadium schwer ausgeprägt, sollte an eine atypische Parkinson-Erkrankung gedacht werden.

\section{Metabolisches Syndrom}

Die herabgesetzte Mobilität aufgrund von Gangunsicherheit und posturaler Instabilität sowie auch kognitive Defizite scheinen bei Parkinson-Erkrankten einen maßgeblichen Faktor für die Entwicklung metabolischer Störungen wie Diabetes mellitus Typ 2 und Hyperlipidämie darzustellen $[25,26]$. Ein erhöhtes Risiko für kardiovaskuläre Ereignisse ist die Folge [27]. Auch besteht ein erhöhtes Risiko für die Entwicklung einer Kardiomyopathie [28].

\section{Osteoporose}

Durch die beeinträchtigte motorische Fähigkeit kommt es zu einer verminderten mechanischen Belastung der Knochen und somit zu einer Einschränkung des Knochenstoffwechsels mit Verstärkung osteokataboler zu Ungunsten osteoanaboler Prozesse [29]. Außerdem fördert die verminderte körperliche Aktivität die Muskelatrophie, und die fehlende Beanspruchung des muskuloskelettalen Systems bewirkt in Kombination mit der ohnehin schon bestehenden parkinsontypischen
Einschränkung der Motorik eine Verstärkung der Gangunsicherheit. Gemeinsam mit der posturalen Instabilität und einer häufig assoziierten autonomen Neuropathie mit gestörter Tiefensensibilität kommt es zu einer stark erhöhten Sturzneigung mit hohem Frakturrisiko.

Die verminderte Leistungsfähigkeit kann zudem mit einer eingeschränkten Sonnenexposition und einem Vitamin-D-Mangel vergesellschaftet sein. Zudem wird vermutet, dass Parkinson-Patienten/-innen eine niedrigere alimentäre Kalzium-Zufuhr haben als gesunde Vergleichsprobanden. Der Zusammenhang besteht möglicherweise in einer Interaktion bei der Aufnahme von L-Dopa und eiweißreicher Kost, die zumeist viel Kalzium enthält. So dürften L-Dopa und Aminosäuren bei der Aufnahme in das Blut und das ZNS um die gleichen Transportmechanismen konkurrieren [30].

\section{Merke \\ All die genannten Effekte haben zur Folge, dass eine deutliche Assoziation zwischen dem Parkinson-Syn- drom und einer verminderten Knochendichte mit damit einhergehendem Frakturrisiko besteht.}

\section{COVID-19}

Die Pflegebedürftigkeit vieler Parkinson-Patienten/-innen macht eine Kontaktlimitierung äußerst schwierig und eine Selbstisolation vielfach unmöglich, und auch Langzeitbetreuende und Pflegende leiden unter der derzeitigen Pandemie. In einer jüngst veröffentlichten Studie wurde der Stellenwert von Komorbiditäten, die mit einem erhöhten Risiko für einen schweren Verlauf einer SARS-CoV-2-Infektion prädisponieren, bei Parkinson-Erkrankten untersucht. Dabei wurde eine hohe Prävalenz ermittelt von

- chronischer Hepatitis B oder C,

- COPD,

- zerebrovaskulären Erkrankungen,

- chronischer Niereninsuffizienz und

- kardiovaskulären Erkrankungen sowie

- insbesondere arterieller Hypertonie und Diabetes mellitus Typ 2.

Letztere weisen in der untersuchten Parkinson-Kohorte quer durch alle aufgeschlüsselten Subgruppen Prävalenzen von etwa $50 \%$ (arterielle Hypertonie) und 20\% (Diabetes mellitus Typ 2) auf.

Merke

Neben diesen Komorbiditäten stützt auch die erhöhte Anfälligkeit für Pneumonien die Hypothese, dass Parkinson-Betroffene eine Prädisposition für einen schweren Verlauf von COVID-19 haben könnten [31]. 


\section{Internistisch relevante Begleitphänomene medikamentöser Parkinson-Therapie}

\section{Orthostatische Dysregulation}

Zahlreiche in der Behandlung des Parkinson-Syndroms verwendete Medikamente können potenziell internistisch relevante Nebenwirkungen auslösen. Hier kann allerdings nicht auf alle Wirkstoffe eingegangen werden, die zur symptomatischen Therapie der unterschiedlichen Manifestationen autonomer Störung zum Einsatz kommen - dies würde den Rahmen des Beitrags sprengen. Das Augenmerk soll vielmehr wiederum auf die Orthostatische Dysregulation gelegt werden, da dieser nicht nur als Leitsymptom der erläuterten atypischen Parkinson-Syndrome, sondern auch als Nebenwirkung vieler in der medikamentösen Behandlung verwendeter Substanzen eine zentrale Rolle zukommt.

- Sowohl L-Dopa als auch Dopamin-Agonisten und NMDA-Antagonisten (wie Amantadin) können zu orthostatischer Intoleranz führen, und zwar vorwiegend in der Anfangsphase nach Einleitung der Therapie.

- Tri- und tetrazyklische Antidepressiva, die zur Behandlung der nicht selten mit Parkinson-Syndromen assoziierten Depression angewandt werden, können (wie der bereits erwähnte Alpha-Rezeptor-Blocker Doxazosin) durch eine Beeinträchtigung der peripheren Vasokonstriktion zu orthostatischer Intoleranz beitragen [23].

\section{Merke}

Hyperhidrose und Seborrhoe können für Erkrankte, die auf transdermal applizierte Medikamente angewiesen sind, ein Problem darstellen und die Verlässlichkeit der Medikamententransfers stören [24].

\section{Langzeitbetreuung}

Durch den degenerativen Charakter der Erkrankung mit allmählich zunehmender motorischer, autonomer, kognitiver und emotionaler Beeinträchtigung der $\mathrm{Pa}$ tienten/-innen stellt Morbus Parkinson eine große Herausforderung dar, und zwar nicht nur für die Betroffenen selber und deren Angehörige, sondern auch für die betreuenden Gesundheitsberufe. Stets sollte ein niederschwelliger und kontinuierlicher Zugang zu medizinischer Versorgung, multimodalen Therapieansätzen und zuverlässigen Informations- und Beratungsangeboten im Sinne einer adäquaten biopsychosozialen Behandlung gewährleistet sein. Eine entscheidende Rolle betrifft hiermit die betreuenden Allgemeinmediziner/innen, die einerseits therapeutisch, andererseits koordinativ wirksam sind und für Betroffene und deren Angehörige Kontinuität im Umgang mit der Erkrankung gewährleisten.

Wesentlicher Bestandteil der Arzt-Patienten-Beziehung sollte hier eine hermeneutische Kommunikation sein, die es dem Betroffenen ermöglicht, selbst Experte seiner Erkrankung zu werden und sich somit aktiv in den Behandlungsplan einzubringen. Mit hermeneutischer Kommunikation ist die angepasst erklärende, deutende Funktion des Gesprächs gemeint, bei der die unterschiedlichen Verstehensmöglichkeiten der Erkrankten Berücksichtigung finden. Nur so können Betroffene und deren Angehörige über Verlauf, Prognose, Therapiemöglichkeiten und mögliche Komplikationen ein Bewusstsein bekommen und dementsprechend agieren.

Enge Zusammenarbeit erfolgt mit Fachärzten/-innen für Neurologie, wo Betroffene zumindest einmal im Jahr zur Verlaufskontrolle sowie ggf. zur Anpassung der Medikation vorstellig werden sollten. Das therapeutische Netzwerk sollte außerdem Ergotherapie, Physiotherapie, ggf. Psychotherapie, Logopädie und in späteren Stadien Pflege durch diplomierte Pflegekräfte umfassen. Innere Medizin, Urologie, Psychiatrie und andere spezialisierte Fachrichtungen runden das Spektrum ab. Wesentlicher Punkt in der Betreuung von Menschen mit Morbus Parkinson sollte in jedem Erkrankungsstadium die Achtsamkeit auf eine höchst mögliche Lebensqualität sein. Die Schwerpunkte in der Behandlung sind dabei immer wieder unterschiedlich zu setzen. 


\section{KERNAUSSAGEN}

- Die frühen Parkinson-Symptome entwickeln sich schleichend, individuell sehr unterschiedlich und sind von Symptomen des normalen Alterungsprozesses oft schwer zu unterscheiden.

- Essenzieller Tremor tritt häufiger auf als eine Parkinson-Erkrankung, betrifft hauptsächlich Hände und Arme beidseits, und nimmt bei Bewegung zu und in Ruhe ab.

- Die Therapie des idiopathischen Morbus Parkinson sollte rechtzeitig, altersgerecht und effizient beginnen und sich nicht ausschließlich auf eine medikamentöse Behandlung beschränken.

- Eine multiprofessionelle Strategie, bei der Hausärzte/-innen eine sehr zentrale Funktion haben können, sollte essenziell sein.

- Vegetative Störungen treten sowohl als Teil der Erkrankung auf als auch in Form von Nebenwirkungen der medikamentösen Therapie.

- Bei frühzeitig auftretenden schweren Störungen des autonomen Nervensystems sollte an ein atypisches Parkinson-Syndrom gedacht werden.

- Bei raschen Veränderungen des klinischen Bildes sollten Infekte, Exsikkose und Nebenwirkungen der medikamentösen Therapie bedacht werden.

- Psychologische Unterstützung ist vor allem in späteren Stadien sowohl für Betroffene als auch für ihre Angehörigen von Bedeutung.

\section{Schlüsselwörter}

Parkinson-Syndrom, Morbus Parkinson, Allgemeinmedizin, Neurodegenerative Erkrankung, Dopaminmangel

\section{Interessenkonflikt}

\section{Erklärung zu finanziellen Interessen}

Forschungsförderung erhalten: nein; Honorar/geldwerten Vorteil für Referententätigkeit erhalten: nein; Bezahlter Berater/interner Schulungsreferent/Gehaltsempfänger: nein; Patent/Geschäftsanteile/Aktien (Autor/Partner, Ehepartner, Kinder) an Firma (Nicht-Sponsor der Veranstaltung): nein; Patent/Geschäftsanteile/Aktien (Autor/Partner, Ehepartner, Kinder) an Firma (Sponsor der Veranstaltung): nein.

Erklärung zu nichtfinanziellen Interessen

Die Autorinnen/Autoren geben an, dass kein Interessenkonflikt besteht.

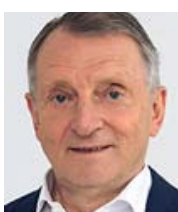

\section{MR Dr. Reinhold Gleh}

Arzt für Allgemeinmedizin, niedergelassener Wahlarzt in Hartberg/Steiermark. Vorstandsmitglied der Steirischen Akademie für Allgemeinmedizin, Lehrbeauftragter an der Medizinischen Universität Graz, Mitglied Akkreditierungsrat Österreichische Akademie der Ärzte, Vorstandsmitglied Österreichische Gesellschaft für Psychosomatik und Psychotherapie in der Allgemeinmedizin, 2010-2015 Präsident der ÖGAM

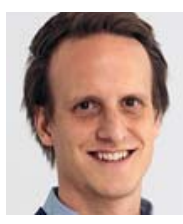

\section{Dr. Tobias Schöberl}

Arzt für Allgemeinmedizin und Facharzt für Innere Medizin an der Abteilung für Innere Medizin des Landeskrankenhaus Hartberg, Notarzt beim Österreichischen Roten Kreuz, Ausbildung in Graz und Hartberg, Madrid sowie Stonetown (Sansibar/Tansania), Schwerpunkte Kardiologie (Rhythmologie und Synkopenabklärung), Endokrinologie (Diabetologie und Knochenstoffwechsel) und spezielle Schmerztherapie

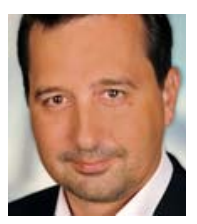

\section{Dr. Werner Seel}

Dr. Werner Seel MSc. MBA. Facharzt für Neurologie, Neurorehabilitation und Sporttherapie; niedergelassener Kassenarzt in Hartberg und Wahlarzt in Graz/Stmk; 2012-2013 Leiter des Bereichs periphere Neurologie/Neuromuskuläre Ambulanz der Univ. Klinik für Neurologie Graz; 2005-2013 OA an der Univ. Klinik für Neurologie Graz; MBA in Health Care and Hospital Management Medizinische Universität Graz; MSc Telemedizin FH Joanneum Graz

\section{Korrespondenzadresse}

\section{Dr. Reinhold Glehr}

Arzt für Allgemeinmedizin

Michaeligasse 12

8230 Hartberg

Österreich

glehr@aon.at

Wissenschaftlich verantwortlich gemäß Zertifizierungsbestimmungen

Wissenschaftlich verantwortlich gemäß Zertifizierungsbestimmungen für diesen Beitrag ist Dr. med. Reinhold Glehr, Hartberg.

\section{IHF-Zertifizierung}

Dieser Artikel wurde gemäß den Kriterien des Instituts für hausärztliche Fortbildung im Deutschen Hausärzteverband (IHF) e.V. zertifiziert. 


\section{Literaturverzeichnis}

[1] Masuhr K, Masuhr F, Neumann M. Duale Reihe Neurologie. 7. Aufl. Stuttgart: Thieme; 2013

[2] Schöls L, Riess O, Krüger R. Zur Genetik und Pathogenese des Morbus Parkinson. Dtsch Arztebl; 1999; 96: Im Internet (Stand 11.10.2020): https://www.aerzteblatt.de/archiv/ 19616/Zur-Genetik-und-Pathogenese-des-Morbus-Parkinson

[3] Diem-Zangerl A, Seppi K, Wenning GK et al. Mortality in Parkinson's Disease: A 20-Year Follow-Up Study. Mov Disord 2009; 24: 819-825

[4] Wenning GK, Kiechl S, Seppi K et al. Prevalence of movement disorders in men and women aged $50-89$ years (Bruneck Study cohort): a population-based study. Lancet Neurol 2005; 4: 815-820

[5] Klebe S. Sekundäre Parkinson-Syndrome. J Neurol Neurochir Psychiat 2014; 15: 69-75

[6] Hassin-Baer S, Sirota P, Korczyn AD et al. Clinical characteristics of neuroleptic-in-duced parkinsonism. J Neural Transm 2001; 108: 1299-1308

[7] Lemke MR, Fuchs G, Gemende I et al. Depression and Parkinson's disease. J Neurol 2004; 251: (Suppl. 06): 24-27 doi:10.1007/s00415-004-1606-6

[8] Bundesministerium für Soziales, Gesundheit, Pflege und Konsumentenschutz. Morbus Parkinson und Demenz. 2020: Im Internet (Stand: 10.10.2020): https://www.gesundheit. gv.at/krankheiten/gehirn-nerven/parkinson/morbus-parkinson-demenz

[9] Richard IH, Schiffer RB, Kurlan R. Anxiety and Parkinson's disease. J Neuropsychiatry Clin Neurosci 1996; 8: 383-392 doi:10.1176/jnp.8.4.383

[10] Kuopio AM, Marttila R], Helenius $\mathrm{H}$ et al. The quality of life in Parkinson's disease. Mov Disord 2000; 15: 216-223

[11] Ellgring $H$, Seiler S, Perleth B et al. Psychosocial aspects of Parkinson's disease. Neurology 1993; 43: S41-\$44

[12] Menza MA, Sage J, Marshall E et al. Mood changes and „onoff" phenomena in Parkinson's disease. Mov Disord 1990; 5 : 148-151

[13] Mollenhauer B, Förstl H, Deuschl G. Demenz mit Lewy-Körpern und Parkinson-Krankheit mit Demenz. Dtsch Arztebl Int 2010; 107: 684-691 doi:10.3238/arztebl.2010.0684

[14] Schrag A, Ben-Shlomo Y, Brown R et al. Young-onset Parkinson's disease revisited-clinical features, natural history, and mortality. Mov Disord 1998; 13: 885-894 doi:10.1002/ mds. 870130605

[15] Klamroth S, Steib S, Devan S et al. Effects of Exercise Therapy on Postural Instability in Parkinson Disease: A Meta-analysis. J Neurol Phys Ther 2016; 40: 3-14

[16] Shen X, Wong-Yu IS, Mak MK. Effects of Exercise on Falls, Balance, and Gait Ability in Parkinson's Disease: A Metaanalysis. Neurorehabil Neural Repair 2016; 30: 512-527

[17] Tomlinson CL, Patel S, Meek C et al. Physiotherapy versus placebo or no intervention in Parkinson's disease. Cochrane Database Syst Rev 2013; 9: CD002817
[18] Psychose bei Morbus Parkinson. Arznei-telegramm 2008; 39: 106-107 (Autorengemeinschaft arznei-telegramm, publiziert am 2. Oktober 2008)

[19] Ahmedani BK. Major Physical Health Conditions and Risk of Suicide. Am J Prev Med 2017; 53: 308-315

[20] Low PA, Opfer-Gehrking TL, McPhee BR et al. Prospective evaluation of clinical characteristics of orthostatic hypotension. Mayo Clinic Proc 1995; 70: 617-622 doi:10.4065/ 70.7.617

[21] Csoti I, Jost WH, Reichmann H. Parkinson's disease between internal medicine and neurology. J Neural Transm (Vienna) 2016; 123: 3-17 doi:10.1007/s00702-015-1443-z

[22] Maeda T, Nagata K, Satoh Y. High prevalence of gastroesophageal reflux disease in Parkinson's disease: a questionnaire-based study. Parkinsons Dis 2013: doi:10.1155/ 2013/742128

[23] Braune S, Lücking CH. Orthostatische Hypotonie. Pathophysiologie, Differentialdiagnose und Therapie. Dtsch Arztebl 1997; 94: 3413

[24] Österreichische Parkinson Gesellschaft. Klinik, Diagnostik und Therapie autonomer Störungen bei Morbus Parkinson und anderen Parkinson-Syndromen. Newsletter 2012; 4: 4

[25] Bohnen NI, Kotagal V, Müller ML. Diabetes mellitus is independently associated with more severe cognitive impairment in Parkinson disease. Parkinsonism Relat Disord 2014; 20: $1394-1398$

[26] Kotagal V, Albin RL, Müller ML. Diabetes is associated with postural instability and gait difficulty in Parkinson disease. Parkinsonism Relat Disord 2013; 19: 522-526

[27] Firoz CK, Jabir NR, Khan MS. An overview on the correlation of neurological disorders with cardiovascular disease. Saudi J Biol Sci 2015; 22: 19-23

[28] Zesiewicz TA, Strom JA, Borenstein AR. Heart failure in Parkinson's disease: analysis of the United States medicare current beneficiary survey. Parkinsonism Relat Disord 2004; 10: 417-420

[29] Downey PA, Siegel MI. Bone biology and the clinical implications for osteoporosis. Phys Ther 2006; 86: 77-91

[30] Abou-Raya S, Helmii M, Abou-Raya A. Bone and mineral metabolism in older adults with Parkinson's disease. Age Ageing 2009; 38: 675-680

[31] Richter D, Bartig D, Krogias C et al. Letter to the editor: risk comorbidities of COVID-19 in Parkinson's disease patients in Germany. Neurol Res Pract 2020; 2: 22https://doi.org/ $10.1186 /$ s42466-020-00069-x

\section{Bibliografie}

Allgemeinmedizin up2date 2021; 2: 39-56

DOI $10.1055 / \mathrm{a}-1220-8145$

ISSN 2699-8696

(C) 2021. Thieme. All rights reserved.

Georg Thieme Verlag KG Rüdigerstraße 14, 70469 Stuttgart, Germany 


\section{Punkte sammeln auf CME.thieme.de}

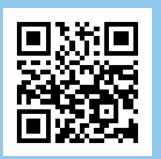

Diese Fortbildungseinheit ist in der Regel 12 Monate online für die Teilnahme verfügbar.

Den genauen Einsendeschluss finden Sie unter https://eref.thieme.de.

Sollten Sie Fragen zur Online-Teilnahme haben, finden Sie unter https://cme.thieme.de/hilfe

eine ausführliche Anleitung. Wir wünschen viel Erfolg beim Beantworten

der Fragen!

Unter https://eref.thieme.de/CXFEMQ7 oder über den QR-Code kommen Sie direkt zur Startseite des Wissenstests.

VNR 2760512021160215236

\section{Frage 1}

Welche Aussage über die Frühsymptomatik des idiopathischen Morbus Parkinson trifft zu?

A Die Symptome sind unspezifisch und vielfältig.

B Die Symptome tretend rasch auf.

C Vegetative Symptome stehen meist im Hintergrund.

D Die Beschwerden im Bewegungsapparat sind in der Regel beidseitig.

E Eine Beeinträchtigung des Geruchssinns tritt selten auf.

\section{Frage 2}

Welches Symptom finden Sie häufig in der Frühphase des Morbus Parkinson?
A beidseitiges Mitschwingen der Arme beim Gehen
B nach vorn gebeugte Haltung
C Seemanns-Gang
D Grimassieren
E Steifigkeit von Armen und Beinen

\section{Frage 3}

Welches Zeichen ist typisch für einen Ruhetremor?
A tritt besonders im Schlaf auf
B beruhigt sich bei Ablenkung
C verschwindet beim Gehen
D verschwindet bei Aufregung
E tritt auf, wenn die Hände nicht gebraucht werden

\section{Frage 4}

Welches ist kein Ausschlusskriterium für einen Morbus Parkinson?
A zerebrale Insulte
B zerebrale Tumore
C posturale Instabilität
D rezidivierende Schädeltraumen
E Neuroleptikamedikation

\section{Frage 5}

Welche psychische Komorbidität tritt selten zusammen mit einem Morbus Parkinson auf?
A Depression
B Manie
C Angsterkrankungen
D Demenz
E Schlafstörungen

\section{Frage 6}

Welche unerwünschte Wirkung hängt mit großer Wahrscheinlichkeit nicht mit einer L-Dopamin-Behandlung bei Morbus Parkinson zusammen?
A Dyskinesien
B On-Off-Phänomen
C Refluxsymptomatik
D Übelkeit
E Serotonin-Syndrom

\section{Frage 7}

Wann ist eine tiefe Hirnstimulation bei Morbus Parkinson kontraindiziert?
A bei einer Demenz
B Leitsymptome sprechen auf Levodopa an
C nichtmedikamentös behandelbare Dyskinesien
D motorische Fluktuationen
E ausgeprägte Rigidität

\section{Frage 8}

Welche nichtmedikamentöse Empfehlung geben Sie einem Patienten bei Problemen mit Freezing?
A Bewegungsrichtung beibehalten
B Ablenkungsbewegungen mit den Armen
C Luft anhalten
D nicht mehr alleine Gehen
E einfach stehen bleiben 


\section{Punkte sammeln auf CME.thieme.de}

\section{Fortsetzung ...}

\section{Frage 9}

Welches Symptom einer autonomen Dysfunktion ist nicht typisch für einen Morbus Parkinson?
A orthostatische Hypotonie
B gastrointestinale Hypomobilität
C hypertone Krisen
D Inkontinenz
E erektile Dysfunktion

\section{Frage 10}

Welcher Faktor kann bei Morbus Parkinson-Erkrankten kaum als osteoporosefördernd gesehen werden?
A Bewegungsarmut
B verminderte Kalzium-Resorption
C Muskelatrophie
D Medikamentennebenwirkung
E orthostatische Dysregulation 